\title{
COMPARISON OF LONG-TIME DELAY IN LASING IN HOMO- AND HETEROEPITAXIALLY GROWN II-VI LASER DIODES
}

\author{
A. Isemann, M. Behringer, H. Wenisch, M. Fehrer, K. Ohkawa \\ AND D. HOMMEL \\ Institute of Solid State Physics, University of Bremen \\ P.O. Box 330 440, 28334 Bremen, Germany
}

\begin{abstract}
Gain guided laser diodes exhibit unexpected low threshold current densities. Under these conditions, lasing only occurs under a current dependent long-time delay, which is three orders of magnitude larger than the time needed to reach population inversion. This effect is attributed to a thermally induced index guiding. The change in temperature of the quantum well region can be estimated using the shift in the wavelength of emission to be up to $70 \mathrm{~K}$. As a further consequence, the threshold current density can be reduced by a factor of 4 simply by changing the pulse width of the applied current.
\end{abstract}

PACS numbers: 42.55.Px

ZnSe laser diodes (LD) grown on GaAs have been investigated for the past six years. The quality of heteroepitaxial devices could be improved to reach lifetimes of up to $400 \mathrm{~h}$ [1]. Recently it has been shown that also ZnSe-substrates offer the potential to realize devices for $\mathrm{cw}$-operation at room temperature [2].

The aim of this paper is to compare the dynamic behavior of heteroepitaxially grown laser diodes to homoepitaxially grown ones. Both investigated samples were double heterostructure separate confinement laser diodes. The heteroepitaxial structure was grown on GaAs-substrate and consists of MgZnSSe claddings of $1000 \mathrm{~nm}, \mathrm{ZnSSe}$ waveguides of $100 \mathrm{~nm}$ to both sides of the quantum well (QW), a $4 \mathrm{~nm}$ thin CdZnSSe quantum well and a conventional $\mathrm{ZnSeTe}$ contact layer. For the homoepitaxial laser diode, an aluminum-doped $\mathrm{ZnSe}$-substrate was used. This diode was identical to the heteroepitaxial device except for the quaternary cladding layer which was lattice matched to $\mathrm{ZnSe}$ and the waveguide which was grown from ZnSe instead of ZnSSe. The CdZnSe quantum well was $4 \mathrm{~nm}$ thin, as well. The contact layers consisted of a low resistivity $\mathrm{ZnSeTe}$-multilayer. Details of growth can be found elsewhere [3, 4]. A palladium-gold contact was evaporated to the $p$-side and $\mathrm{Al}_{2} \mathrm{O}_{3}$ was used as insulating material to form stripe widths of $10 \mu \mathrm{m}$ and $20 \mu \mathrm{m}$, respectively. No etching beside the contact was performed. The lasers with a cavity length of $590 \mu \mathrm{m}$ and $755 \mu \mathrm{m}$, respectively; were mounted episide up, i.e. with the substrate side onto copper heat sinks. 


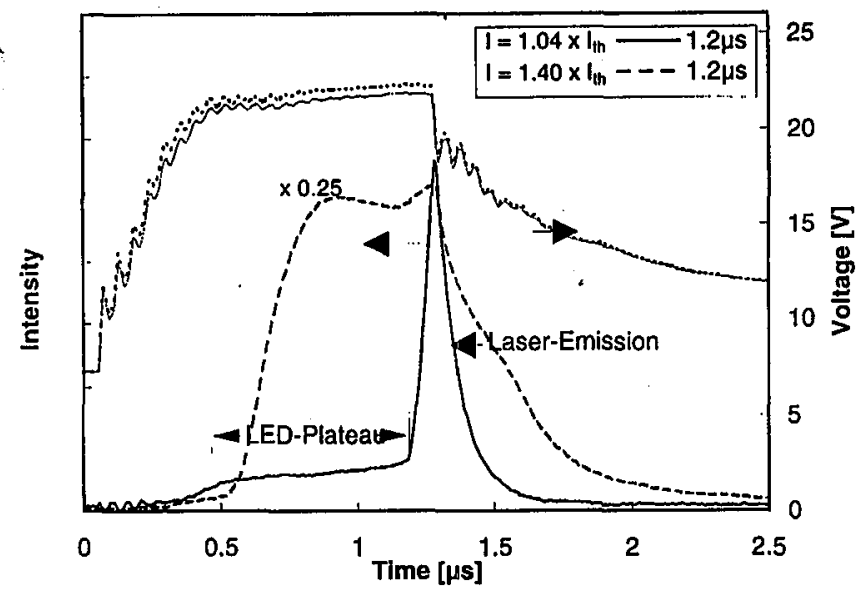

Fig. 1. The time-resolved intensity (arb. units) during the pulse shows a delay in the onset of lasing in a heteroepitaxial laser diode. With increasing current this delay is reduced.

These laser diodes were operated in pulsed mode at varying pulse widths but with a constant duty cycle of $0.1 \%$. The time-resolved intensity of the light over the applied current pulse was investigated using a photodiode and a digital oscilloscope. In Fig. 1 typical pulse shapes for the heteroepitaxial laser are depicted. There is an initial delay for about $200 \mathrm{~ns}$ before low intensity emission in LED-mode is detected. This delay is caused by a slow build-up of the applied voltage and thus of the current which is due to the power supplies characteristics. During the period labeled LED-plateau, the diode is operating in LED-mode. One can clearly distinguish this LED-emission from the stimulated emission after the steep increase in intensity at about $1.2 \mu \mathrm{s}$. To reach population inversion, a typical timescale of nanoseconds is needed [5]. The observed delay of about 1-2 $\mu \mathrm{s}$ is three orders of magnitude larger. This delay can be explained by thermally induced index guiding.

The heat generated at the $p$-contact and in the path of the current through the layers increases the refractive index. Thus, a change of the refractive index in a plane of the quantum well underneath the contact stripe is produced resulting in a better confinement of the wave. The overlap between the inverted region of the QW and the guided wave is increased as well as the amplification, at the same current density.

The temperature, needed to produce an index step which is large enough to confine the wave sufficiently, needs some time to propagate from the contact to the QW. This explains the delay. During the time while the QW is being heated, until the index step is sufficient to confine the wave enough, therefore the overlap with the inverted region of the QW yields enough amplification to overcome the losses, the device is operating in LED-mode. When index-guiding is established, stimulated emission is supported and the light intensity increases drastically. It can also be seen in Fig. 1 that by increasing the current density not only the 


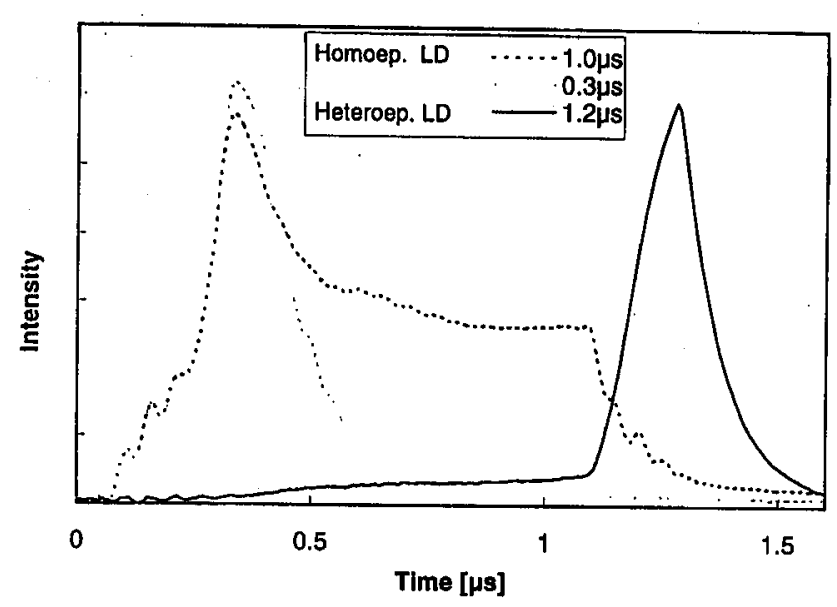

Fig. 2. The time-resolved intensity (in arb. units) during a pulse for a homoepitaxial laser. For comparison, the pulse shape of a heterostructure laser is also depicted.

peak intensity of the light rises, but also the delay time is reduced. This can be understood when considering that at a higher current density more heat is generated at the $p$-contact, thus the same change in temperature at the $\mathrm{QW}$ is reached earlier.

In contrast, the homoepitaxial laser shows a completely different behavior. In Fig. 2 two typical pulse shapes are depicted. The laser emission starts at the beginning of the current pulse after a short delay of $0.2 \mu \mathrm{s}$ and the intensity decreases towards longer times. The short delay at the turn on and the decrease in intensity after the initial high intensity peak during stimulated emission remains even for longer pulse widths. For higher current densities, only a small change of the delay can be observed (not shown) and even at very high current densities a decrease after the lasing period is detected. For comparison a typical pulse for a heterostructure laser is drawn in the same figure. Another difference is the threshold current density which amounts to $970 \mathrm{~A} / \mathrm{cm}^{2}$ for the homoepitaxial LD. This is higher than for the heteroepitaxial laser diode with $800 \mathrm{~A} / \mathrm{cm}^{2}$ at $2 \mu \mathrm{s}$, where one also has to consider the deeper quantum well for the homoepitaxial device. In this laser structure, the thermal index step is not sufficient to confine the wave in such a way that lasing occurs at low current densities. This is attributed to the thinner dielectric waveguide and claddings compared to the other structure. In that case, the wave extends into the $p$-contact, which has a smaller band gap than the $\mathrm{QW}$ and thus strongly absorbs the wave.

The temperature of the QW can be estimated from the change of emission wavelength. In the heterostructure this shift amounts to $6.5 \mathrm{~nm}$ which corresponds to about $50^{\circ} \mathrm{C}$ when going from $0.1 \mu \mathrm{s}$ to $5 \mu$ s pulses. Most of the temperature increase occurs while changing the pulse width from $0.1 \mu \mathrm{s}$ to $1 \mu \mathrm{s}$. The homoepitaxial laser diode shows no shift in the emission wavelength when changing the pulse width from $0.3 \mu \mathrm{s}$ to $2 \mu \mathrm{s}$, due to the laser emission occurring only during the same time period only at the beginning of the pulse. 


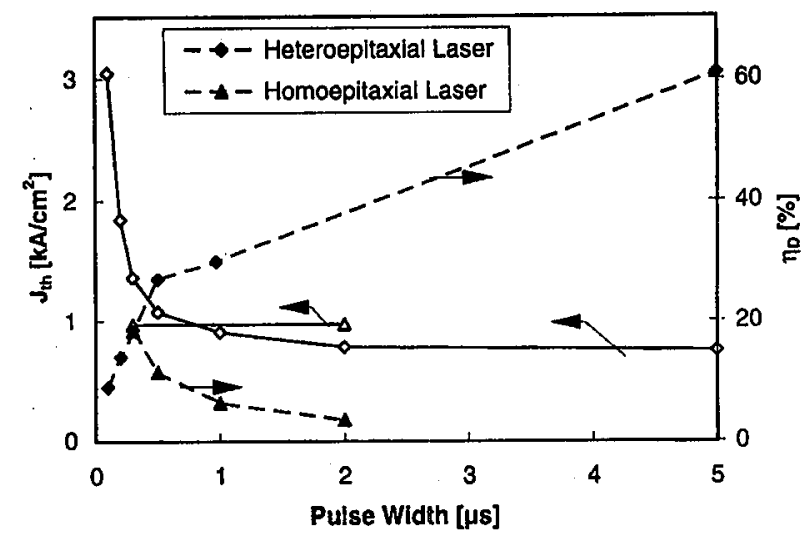

Fig. 3. Threshold current densities and quantum efficiencies in dependence of the pulse width.

The increase in the confinement factor also shows up in the differential quantum efficiency, which rises for longer pulses, as can be seen in Fig. 3. The time needed to build up the thermal index step also influences the threshold current density. In Fig. 3 the threshold current density vs. pulse width is plotted. Most of the reduction occurs within 1-2 $\mu$ s for the heteroepitaxial device, thus most of the index change to confine the wave is built up within 1-2 $\mu \mathrm{s}$. At long pulses, the threshold is lower by a factor of four compared to short pulses. For longer times, the $\mathrm{QW}$ region reaches a higher temperature due to longer heating resulting in a higher index step and thus a better confinement. Although less current produces less heat, the increase in time for the heat to transfer to the $\mathrm{QW}$ is the dominant factor. The homoepitaxial diode on the other hand shows almost no such dependence of threshold current density. This can be understood when considering that the lasing action occurs at the same time during the pulse being independent of the pulse width. At this time, the temperature in the QW is approximately the same resulting in a fixed wavelength when going from $0.3 \mu$ s to $2.0 \mu$ s pulse widths.

\section{References}

[1] E. Kato, H. Noguchi, M. Nagai, H. Okuyama, S. Kijima, A. Ishibashi, Electron. Lett. 34, 282 (1998).

[2] F. Nakanishi, H. Doi, N. Okuda, T. Matsuoka, K. Katayama, A. Saegusa, H. Matsubara, T. Yamada, T. Uemura, M. Irikura, S. Nishine, Electron. Lett. 34, 496 (1998).

[3] H. Wenisch, K. Ohkawa, A. Isemann, M. Fehrer, D. Hommel, Electron. Lett. 34, 891 (1998).

[4] K. Ohkawa, M. Behringer, H. Wenisch, M. Fehrer, B. Jobst, D. Hommel, M. Kuttler, M. Strassburg, D. Bimberg, G. Bacher, D. Tönnies, A. Forchel, Phys. Status Solidi B 202, 683 (1997).

[5] M. Ozawa, A. Egan, A. Ishibashi, Solid State Commun. 94, 87 (1994). 